\title{
Topics
}

\section{Possible Utilization of the Laser Energy Transmission in Space}

\author{
By Nobuki Kawashima, Kazuya TaKeda and Kyoichi Yabe \\ Liaison Center, Kinki University, Higashi-Osaka, Japan
}

(Received June 27th, 2011)

\begin{abstract}
Using the laser energy transmission technology, we have succeeded to fly a small airplane and a helicopter for a long time ( infinite time in principle). Extrapolating the technology, it can be concluded that the technology is available for the near earth space mission in which we cannot use the solar energy such as the ice exploring rover in the bottom of the crater on the lunar polar region. For the application to a deep space mission is challenging, and looking the progress of LISA project (NASA-ESA), it is not impossible.
\end{abstract}

Key Words: Laser, Energy Transmission, Lunar Ice, Small Aircraft

\section{Introduction}

Where and when will be the wireless energy transmission required in space? In most cases in near earth environment, the solar energy is available, and the simplest way to acquire the energy is to use the solar energy. The wireless energy transmission of energy is required when the solar energy is not available.

One possibility is the supply of the energy to the area in the long night on the lunar surface ${ }^{1)}$. Another possibility is the bottom of the crater in the lunar polar region, where it is expected that the ice exists. If it is real and the amount is large, it is certain that the construction of the lunar base will be accelerated. Nearly a decade ago, one of the author proposed that it is important to develop a scientific rover to explore the presence and the amount of the ice in the lunar polar region using the laser transmission technology (Fig. 1) ${ }^{2)}$.

The recently proposed application is on the Space Elevator 3). More future challenging application is in the deep space mission beyond Mars, where the solar energy density is not sufficient. So far, RTG (Radioisotope Thermoelectric Generator) has been used in the past missions such as Voyager, Galileo etc, however, RTG will not be allowed to use from the safety standpoint against the earth environment if other safer means is available.

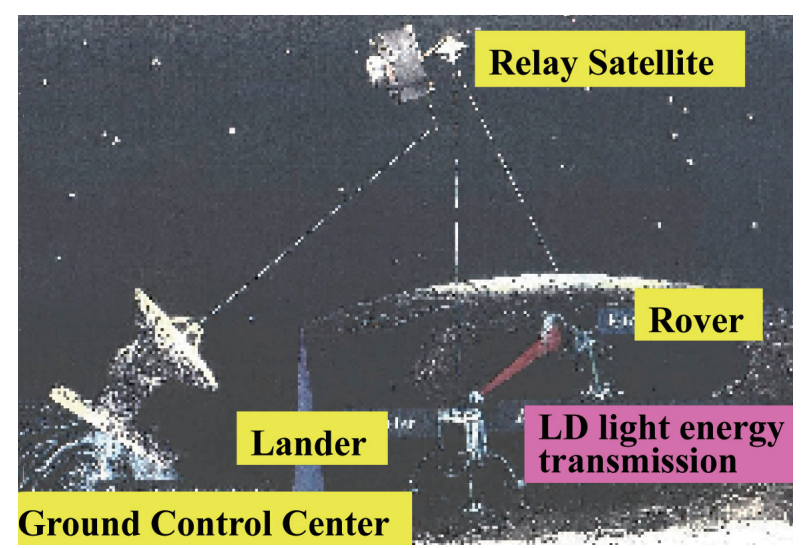

Fig. 1. Rover to explore to confirm the presence of ice on the moon using the laser energy transmission.

\section{Past Experiments}

\subsection{Lunar rover development}

Since the bottom of the crater is perpetually dark and no sun light is available, in order to activate an exploring rover there, we cannot use the solar energy. What we proposed is that since the periphery of the crater is all days sunlit, the solar energy is first converted to the electricity and then to the laser. The laser is transmitted to the rover and there it is converted to the electricity through a solar panel to drive the rover. ${ }^{4)}$

We have constructed a full size rover and tested successfully a $1.2 \mathrm{~km}$ transmission on the ground. (Fig 2) ${ }^{5}$

This idea has not been realized as a space mission yet, and recognizing that it is necessary that the technology which can be used in space shall be first used and well established on the ground. We successfully developed a system to fly small aircrafts using the laser energy transmission technology.

The application of this laser driven aircrafts we aim is to use it as a surveillance flight over the area where the human access is difficult in the case of natural disasters such as floods or earthquakes. A long flight can be realized and no return of the aircraft to the base for the refueling or recharging is needed. More important occasions in which the laser energy transmission is indispensable are in serious nuclear power plant accidents or terrorism disaster site using chemical or biological weapons. Rescue robots cannot return for the energy refueling because of heavy contamination.

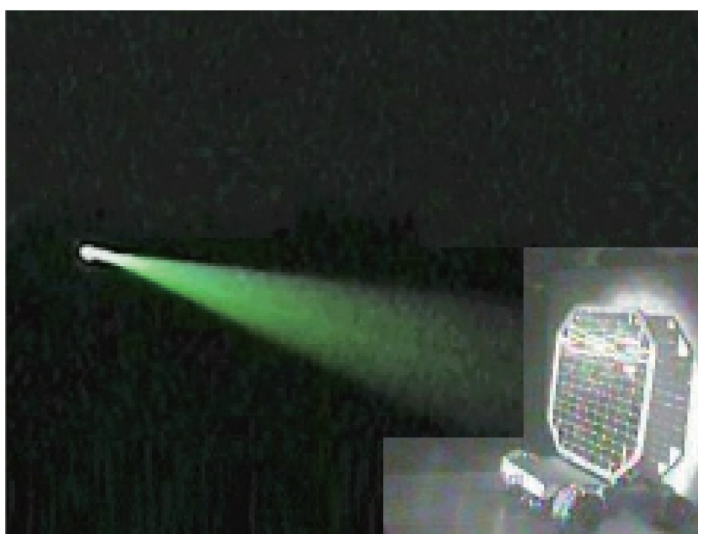

Fig. 2. $1.2 \mathrm{~km}$ laser energy transmission experiment. The laser coming from the center-left is irradiated to the lunar rover model (bottom-right). 


\subsection{Small airplane flight}

We selected a small kite-plane as the first spacecraft (Fig. 3). The laser we used is a fiber-fed semi-conductor laser. The laser excited by laser diode is coming out from a fiber of the core size of $400 \mu \mathrm{m}$. The total power we use was $350 \mathrm{~W}$. It was focused on a solar panel of about $30 \mathrm{~cm}$ in diameter which was equipped under the kite-plane. The tracking is very important and we have realized a scheme in which a reflected laser light from a corner reflector placed in the center of the panel is used and it is focused on a 4-element diode. The direction of the laser beam is controlled so that the laser light shall come back in the very center of the 4-element diode.

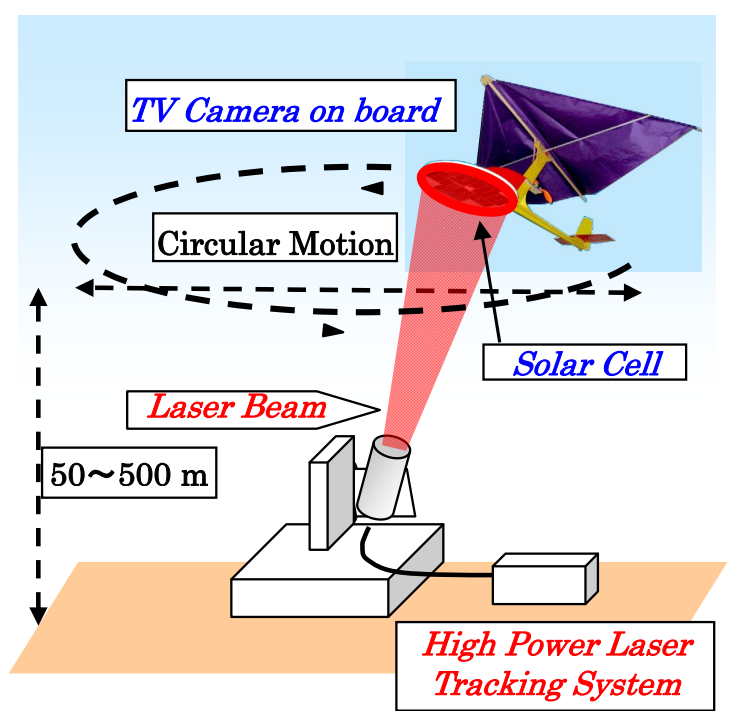

Fig. 3. System configuration of a kite plane driven by a laser energy transmission.

We tested in a baseball dome stadium successfully in 2006 at an altitude of $50 \mathrm{~m}$ and with the circulating radius of about 10 $\mathrm{m}$. The tracking accuracy of $0.2 \mathrm{mrad}$ and the tracking speed of $10 \mathrm{~m} / \mathrm{sec}$ at $50 \mathrm{~m}$ high have been accomplished. (Fig. 4) ${ }^{6,7)}$

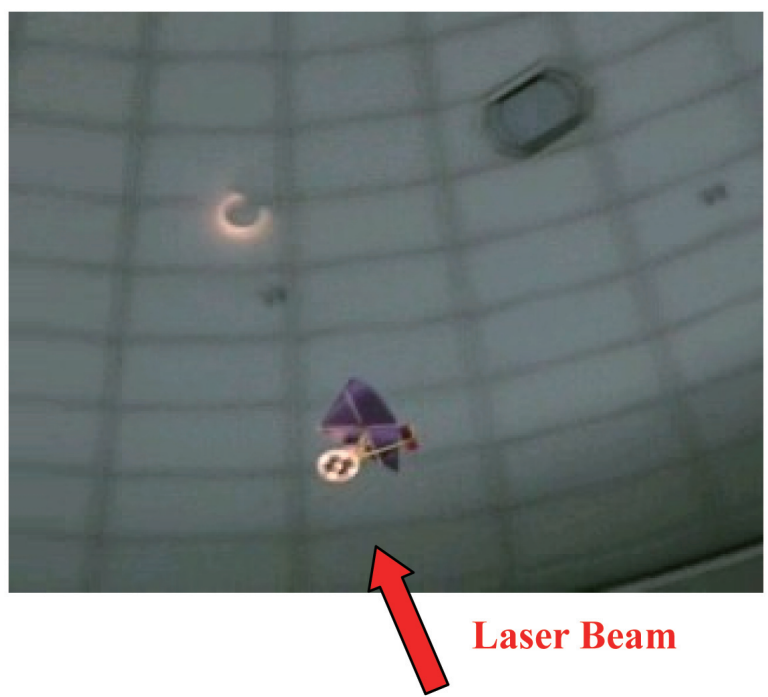

Fig. 4. TFlying kite-plane under the auto-Tracking (The solar panel is irradiated by the laser light).
In order for this system to be used in real disaster cases, the horizontal range shall be increased, however, in the present system, to increase the horizontal range, the vertical altitude shall also been increased. To reduce the vertical altitude, the solar panel shall be set vertically, then the attitude control of the solar panel conflicts with a stable spacecraft maneuver.

\subsection{Helicopter flight}

To solve this problem, the helicopter is the most appropriate and we can set the solar cell panel vertically (Fig.5). However, the helicopter requires much more electric power than the airplane.

One solution is to increase the laser power, then we need a more efficient cooling of the solar panel.

The other solution is to increase the conversion efficiency from the laser power to the electricity.

For the former, we have increased the laser power up to 530 $\mathrm{W}$. The cooling of the solar panel is accomplished by setting it under the body as shown in Fig. 5 .

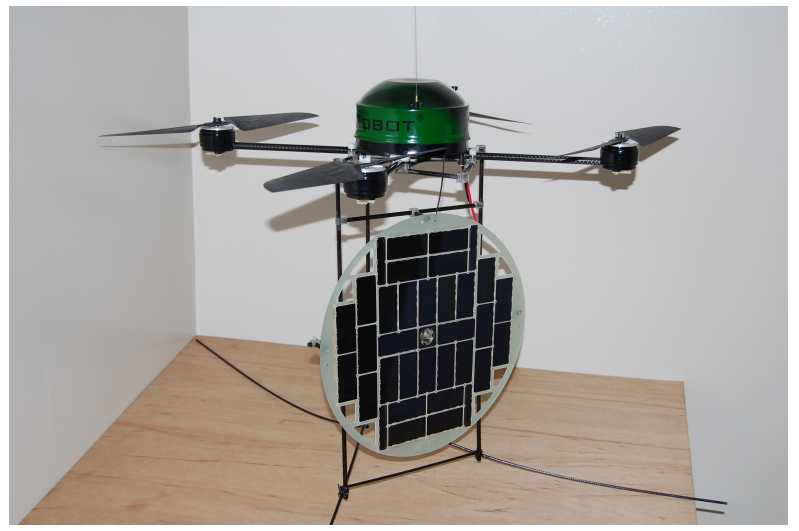

Fig. 5. Helicopter hanging a solar cell panel under it.

For the latter, first we filled the panel with the solar cell as much as possible. Since the shape of the solar cell commercially available is fixed, we tried to cut it in half. More improvement of the efficiency has been achieved by optimizing the cell connection. The detail of this improvement will be described elsewhere.

It has taken a long time trial and error procedure and finally we have realized a stable long time flight in 2008. We use a small battery to maintain the safe landing of the helicopter even when the proper tracking of the laser receiving solar cell panel fails. We have confirmed that even after more than one hour flight, the battery maintains a fully charged ${ }^{8}$. (Fig. 6)

\subsection{Space elevator program}

Most recent development of high power laser energy transmission application is on the space elevator program - International contests have been conducted supported by NASA $^{9)}$.

\section{Future Prospect for the Application in Space Propulsion}

\subsection{Laser}

The laser diode is suitable for the laser energy transmission 


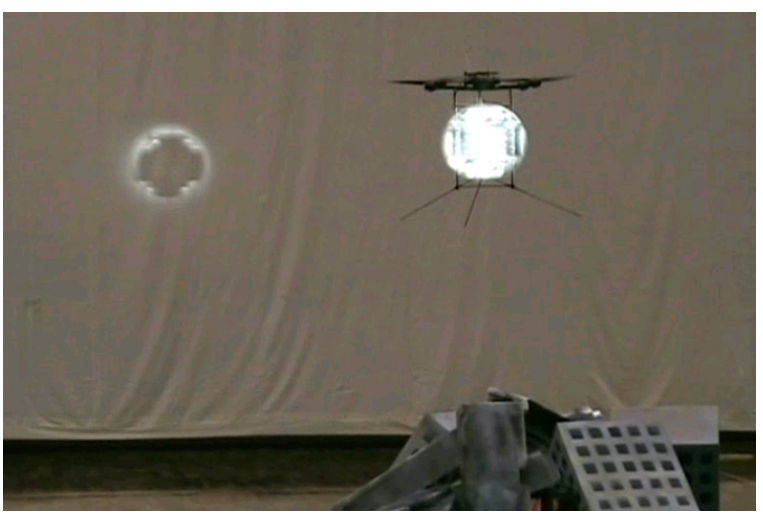

Fig. 6. Helicopter flight over a simulated disaster ruin receiving the energy in the laser energy transmission system A.

use : small and light weight, high efficiency, very simple and easy to handle etc. We only used $200 \mathrm{~W}$ units in parallel, however, now multi-kW class of fiber-output type is available. Consequently, for a short distance application such as the lunar ice exploring rover, the laser diode is sufficient.

For much longer distance transmission, the recent development of the fiber laser will be better. The fiber-fed laser diode system is only a very strong light source with a high brightness but without coherent characteristics as the laser. Therefore, the optical system follows a simple geometric optics. The fiber laser has a better characteristic as the laser and the core size is much smaller.

More innovative attempt is the direct conversion of the solar radiation to the laser.

When we use a laser, it is common that the solar radiation is converted to electricity and the laser is excited by using the electricity. Then the conversion efficiency cannot be high and it is very difficult to obtain the overall conversion efficiency more than $15 \%$.

The direct conversion of the solar energy to laser has been developed in ILT (Inst. Laser Technol.) in Osaka for a decade. Recent achievement is the conversion efficiency more than $40 \%{ }^{10)}$

\subsection{Transmission Range}

So far, we have tested up to $1.2 \mathrm{~km}$ and so long as the operation with a precise tracking is concerned, we have tested only up to $50 \mathrm{~m}$, tracking accuracy of $200 \mu \mathrm{rad}$ and tracking speed of $0.2 \mathrm{rad} / \mathrm{sec}$.

It is not difficult to improve to $10 \mathrm{~km}$ range, tracking accuracy of $\mu \mathrm{rad}$ as an extension of the present technology. Therefore, the application to the lunar rover mission is already within the capable technology.

As to the application to the deep space mission, it is quite challenging. The technology to achieve the tracking accuracy of prad ( pico-radian : $10^{-12} \mathrm{rad}$ ) will be required. One may feel that such a large jump of $\mu \mathrm{rad}$ - to - prad is impossible, however, the sub-nrad technology is already going on.

NASA-ESA joint mission of LISA ( Laser Interferometer Space Antenna) is developing the target of sub-nrad accuracy ${ }^{11)}$. LISA is an attempt to detect a low frequency gravitational wave of Einstein's general relativity generated by large ultra- high density object activities in space. A triangle interferometer of the arm length of 5 million $\mathrm{km}$ is to be constructed in the sun orbit of 1 AU radius ${ }^{11)}$. Though another 2 orders of magnitude improvement is required, but it will not be impossible.

\section{Conclusion}

The application of the laser energy transmission technology to the propulsion of small aircrafts on the ground and the lunar ice rover is described. The technology of the short distance application on the ground and in near earth space is already well matured and can be used at any time.

The application of this technology to the deep space mission is not in the area which can be realized very easily, however, it is worth challenging.

This work has been supported by the Collaboration with Local Communities" Project by MEXT.

\section{References}

1) Landis, G. A.: Moon night power by laser illumination, AIAA Journal of Propulsion and power, 8 No. 8 (1992).

2) Kawashima, N.: "The importance of the development of a rover for the direct confirmation of the existence of ice on the moon, "Trans. Japan. Soc. Aeronaut. Space Sci. 43, (2000), pp.34-35.

3) http://en.wikipedia.org/wiki/Space_elevator

4) Takeda, K., Tanaka, M., Miura, S., Hashimoto, K. and Kawashima, N.: "Laser power transmission for the energy supply to the rover exploring ice on the bottom of the crater in the lunar polar region, "SPIE, 4632, (2002), pp.199-223

5) Kawashima N. and K. Takeda, " $1.2 \mathrm{~km}$ laser energy transmission for the development of a lunar rover confirming the presence of ice on the moon, " Proc. ILC 2003 / ILEWG 2004.

6) Kawashima, N., Takeda, K. and Yabe, K.: "Application of the laser energy transmission technology to drive a small airplane" Chinese Optics Letters 5 Suppl. (2007) S109-110.

7) Takeda, K., Kawashima, N. and Yabe, K.: "Laser Energy Transmission to a Small-Unmanned Aerial Vehicle" (in Japanese) Uchu Gijutsu 7 (2008) pp.27-32.

8) Kawashima, N. "Laser Energy Transmission" (in Japanese) IEEJ Journal 129 (2009) pp.422-425.

9) http://www.spaceward.org/elevator2010-pb

10) Saeki, T., K. Imasaki, and S. Nakatsuka, Review of the direct conversion of the solar energy to laser(in Japanese) Rev. Laser Eng. 37 (2009) pp. 120.

11) R. Stebbins et al., "Laser Interferometer Space Antenna (LISA) : A Response to the Astro2010 RFI for the Particle Astrophysics and Gravitation Panel 2009. 